\title{
Influence of the simplifications applied to the surrounding gas composition of evaporating droplets in air-blown and oxy-fuel combustion atmospheres
}

\author{
Fernando Luiz Sacomano Filho*1, Jeroen Adrianus van Oijen², Luis Eduardo de Albuquerque \\ Paixão e Freire de Carvalho ${ }^{1}$, Guenther Carlos Krieger Filho ${ }^{1}$ \\ ${ }^{1}$ Department of Mechanical Engineering, Escola Politécnica da Universidade de São Paulo, \\ São Paulo, Brazil. \\ ${ }^{2}$ Department of Mechanical Engineering, Technische Universiteit Eindhoven, Eindhoven, \\ Netherlands. \\ *Corresponding author email: fernando.sacomano@usp.br
}

\begin{abstract}
Droplets evaporating in a combustion environment usually face a strong variation of the surrounding gas composition during their respective lifetimes. Studies have been demonstrated that different approaches applied to represent the surrounding gas composition do interfere with the prediction of evaporation rates, mixture properties, and, consequently, flame characteristics as the propagation speed. Effects of gas phase modeling may change when the atmosphere is less diluted with inert (or less reactive) species as in oxy-fuel combustion atmospheres, where the oxidant is depleted in nitrogen. Considering air-blown and oxy-fuel combustion atmospheres, this work proposes consistent modeling simplifications to be applied to droplet evaporation computations. To accomplish this task, numerical simulations of flames propagating in droplet mists of water are conducted in a one-dimensional framework considering a detailed description of the chemistry. Analysis evolves gradually in a systematic fashion, considering the full description of the chemistry in properties description up to the most simplified approaches. It is found that the flame speeds vary by $10 \%$ for the different approximations. Results summarize effects of the different applied simplifications and indicate their impact in simulations of combustion processes.
\end{abstract}

\section{Keywords}

Droplet evaporation; oxy-fuel; combustion; spray; detailed chemistry.

\section{Introduction}

Heat and mass transfer on droplets do strongly depend on the carrier gas composition [8, $14,17,18]$. While the accurate and detailed description of the gas composition is not a trouble in non-reactive atmospheres, it becomes a challenge in combustion processes. On one hand, combustion processes may involve hundreds of species which may interact in a complex fashion following thousands of intermediary reactions. On the other hand, detailed information about the mixture composition is seldom available in numerical simulations of combustion processes, as simplified chemistry models are often applied. With this aspect in mind, recent works have concentrated efforts on proposing different approaches to improve the description of surrounding gas composition of evaporating droplets in combustion simulations. Preliminary analyses conducted in [13] indicated that simplifications of the surrounding gas composition are able to interfere not only with the evaporation rates, but also with the prediction of flame propagation speeds. This study was deepened in [14], where a novel strategy to represent the surrounding gas properties of droplets evaporating in a combustion environment is presented. Different approaches have been also proposed recently in [2, 5, 6, 16]. Those presented in [5], [6], and [2] are developed in the context of tabulated chemistry, while the strategy presented in [16] has no limitations to chemistry models as the one proposed in [14]. A common characteristic to all of these previous works refers to the consideration of fuel droplets, in which the 
evaporation process is responsible to deliver fuel vapor to the combustion reactions. Another common aspect refers to the focus on the air-blown process, where air is used as an oxidizer. An exception occurs in [6], where Moderate or Intense Low-oxygen Dilution (MILD) combustion is considered. During the development of [10], where methane oxy-fuel flames diluted with water droplets were studied, it was noticed that the performance of simplifications applied to represent the surrounding gas composition differ from air-blown to oxy-fuel processes. Additionally, the consideration of a combustion flue gas species as the liquid vapor requires a special care when using previously proposed models, for instance the one presented in [14].

In view of the previously listed aspects, this work investigates and proposes consistent modeling simplifications to represent the surrounding gas composition of water droplets in air-blown and oxy-fuel combustion atmospheres. Accordingly, numerical simulations of flames propagating in droplet mists of water are performed in a one-dimensional framework considering a detailed description of the chemistry. Analysis evolves gradually in a systematic fashion, considering the full description of the surrounding mixture composition up to the most simplified approaches. This work can be seen as a continuation of both [14] and [10], as it complement the modeling strategies proposed in [14] and improve the computational efficiency of water diluted oxy-fuel combustion from the methodology applied in [10].

\section{Material and methods}

Analysis were conducted with the CFD code CHEM1D [19], which has been extended with a Lagrangian module in [15] and gradually enhanced in [14] and [10] to investigate flames propagating in fuel and non-fuel droplet mists. Herein, two-phase flow simulations are performed within a Eulerian-Lagrangian framework, in which a full inter-phase two-way coupling is considered. Following this modeling strategy, the simulations presented in this manuscript mimic unstrained laminar flames propagating in mono-dispersed and isotropic droplet mists. In order to isolate the diverse underlying phenomena (as done in $[9,14,15]$ ), the following simplifications and approximations are considered: (a) the multi-dimensional aspects of the droplets dispersion does not modify the flame surface, (b) mists are diluted, thereby no interaction among droplets is accounted for, (c) no micro-mixing model is included, so that all the mass evaporated from a drop instantaneously fills the host control volume. Additionally, when a droplet crosses a cell boundary a splitting factor is used to interpolate the source of vapor between the two host control volumes. In our Lagrangian algorithms, parcels are tracked instead of real drops. In this way, a parcel may represent more or even less than one real drop. The role played by them can be illustrated as a dispersed source of water vapor which follows the physical models described in the two following sections. For more details about the numerical methods and simplifications applied to the dispersed phase the reader is referred to [14, 15].

\section{Gas Phase}

The description of the carrier phase follows a variable-density low Mach number formulation. Following the strategies presented in $[10,15,19]$, the set of equations employed here is

$$
\begin{aligned}
& \frac{d \dot{m}}{d x}=S_{\mathrm{V}}^{L}, \\
& \frac{d\left(\dot{m} Y_{i}\right)}{d x}-\frac{d}{d x}\left(\rho Y_{i} V_{i}\right)=\dot{\omega}_{i}+\delta_{i k} S_{\mathrm{V}}^{L}, \quad \text { with } \quad Y_{i} V_{i}=-D_{i, m}\left[\frac{d Y_{i}}{d x}+\frac{Y_{i}}{\bar{M}} \frac{d \bar{M}}{d x}\right]-\frac{D_{i}^{T}}{\rho T} \frac{d T}{d x} \text {, and } \\
& \frac{d(\dot{m} h)}{d x}=\frac{d}{d x} q+S_{\mathrm{h}}^{L} \quad \text { with } \quad q=-\lambda \frac{d T}{d x}+\rho \sum_{i=1}^{N} Y_{i} V_{i} h_{i}-R T \sum_{i=1}^{N} \frac{D_{i}^{T}}{X_{i} M_{i}} \frac{d X_{i}}{d x} .
\end{aligned}
$$

Equation 1 ensures the mass conservation of the coupled system, where $\dot{m}=\rho u$ is the mixture mass flux, $x$ the spatial coordinate, and $S_{\mathrm{V}}^{L}$ the source term of vapor. The transport of chemical 
species is described by Eq. 2 . Herein, $Y_{i}$ is the mass fraction of the species $i \in\left[1, N_{s}-1\right], N_{s}$ total number of participating species, $V_{i}$ is the diffusion velocity, $\dot{\omega}_{i}$ the reaction rate, and $\delta_{i k}$ the Kronecker delta. The subscript $k$ in Eq. 2 refers to the vapor species. The last equation of this set is associated with the conservation of energy, which is expressed in terms of the absolute enthalpy $h$. The absolute enthalpy of each transported species is represented by $h_{i}$, while the coupling term between phases is denoted by $S_{\mathrm{h}}^{L}$. For details about the coupling source terms, the reader is referred to [15]. Finally, $q$ corresponds to the heat flux in which $\lambda$ the thermal conductivity, $T$ the temperature, $R$ the gas constant, $D_{i}^{T}$ the thermal diffusion coefficient, $X$ molar fraction, and $M$ molar mass. The last term on the RHS of $Y_{i} V_{i}$ equation (see Eq. 2) includes Soret effects on mass transport. Within this approach, diffusion coefficients $\left(D_{i, m}\right.$ and $D_{i}^{T}$ ) are estimated following the strategy proposed by Ern and Giovangigli [4].

\section{Liquid Phase}

In the one-dimensional Lagrangian framework, the tracking of a parcel is essentially described by two ordinary differential equations (ODE) (e.g. Sirignano [18])

$$
\frac{d x_{p}}{d t}=u_{p}, \quad \text { and } \quad \frac{d u_{p}}{d t}=\frac{3}{4} \frac{C_{D}}{d_{p}} \frac{\rho}{\rho_{p}}\left|u-u_{p}\right|\left(u-u_{p}\right),
$$

for the parcel position and acceleration, respectively. In the present context, drag is the unique force acting on a droplet. For both equations, the subscript $p$ indicates quantities of droplet parcels. Specifically, $x_{p}$ corresponds to the droplet position, $\rho$ is the density, $u$ the velocity, $t$ is time, and $d_{p}$ the droplet diameter. To compute the drag coefficient $C_{D}$ the model recommended by Yuen and Chen [20] is adopted.

The structures of heat and mass transfer equations follow those proposed by Miller et al. [8], which are implemented in Chem1D [15]. Within this formulation, the Abramzon-Sirignano model (see [1]) is adopted in the present study. Accordingly, both equations are given by

$$
\frac{d T_{p}}{d t}=\frac{f_{2} N u}{3 \operatorname{Pr}}\left(\frac{\theta_{1}}{\tau_{p}}\right)\left(T-T_{p}\right)+\left(\frac{L_{V}}{c_{l}}\right) \frac{\dot{m}_{p}}{m_{p}}, \quad \text { and } \quad \frac{d m_{p}}{d t}=-\frac{S h}{3 S c}\left(\frac{m_{p}}{\tau_{p}}\right) H_{M},
$$

with $T$ the temperature, $N u$ the Nusselt number, $f_{2}=-\dot{m}_{p} /\left(m_{p} B_{T}\right)\left[3 \tau_{p} \operatorname{Pr} / N u\right]$ a correction factor due to evaporation, $\operatorname{Pr}$ the Prandtl number, $L_{V}$ the heat of vaporization, $\theta_{1}=\left(c_{p, V} / c_{l}\right)$, $c_{l}$ the specific heat of the liquid, $\tau_{p}=\rho_{p} d_{p}^{2} / 18 \mu$ expresses the particle relaxation time, and $\dot{m}_{p}=d m_{p} / d t$. In Eq. 5, $S h$ is the Sherwood number, $S c$ the gas Schmidt number, and $H_{M}$ represents the specific driving potential for mass transfer $\left(H_{M}=\ln \left[1+B_{M, e q}\right]\right)$. The nondimensional numbers $\operatorname{Pr}$ and $S c$ are explicitly computed at each time integration of the evaporation equations based on material properties of the mixture film surrounding each tracked droplet. Notice that both are also used to compute the $L e=S c / P r$, which is consequently employed for instance in heat transfer Spalding number calculation (e.g. [14]). Within equations presented for $f_{2}, \theta_{1}$, and $H_{M}, c_{p}$ is the specific heat at constant pressure, $B_{T}$ and $B_{M}$ denote the Spalding transfer numbers for heat and mass, respectively. Subscripts ${ }_{G}$ and ${ }_{V}$ correspond to surrounding gas properties and vapor properties, while $e q$ refers to properties evaluated with the assumption of phase equilibrium. Observe that heat transfer by radiation is not considered in dispersed phase computations.

An important aspect is that the carrier phase is described in a steady-state formulation, while the dispersed phase follows an unsteady framework. To couple both approaches, the methodology proposed in [15] is applied here.

The surrounding gas properties exclusively used in Lagrangian subroutines (i.e. $\mu, \lambda, c_{p}$, and $D_{i j}$ ) are key quantities for the proposed approach in the present work. Herein, we follow the strategy detailed in [14], where the gas composition in the far field follows the species diluted (SD) approach. Briefly, the proposed approaches in [14] rely on the concept that, when an insufficient number of species is available to detail the mixture composition of the carrier gas, 
a specific species is used as a "filling gas" to attain that $\sum_{i}^{N} Y_{i}=1$. This is a typical problem in tabulated chemistry simulations, where only a few species are available in order to save memory $[2,6,14]$. Within this context, vapor species is one of the few that must be available for the computation of droplet evaporation, where the remaining gas mixture is often represented by air [11, 12]. Both approaches presented in [14] overcomes such a simplification by including a few more species to represent the surrounding gas properties. However, when considering oxy-fuel combustion diluted with liquid water, a conceptual discrepancy is noticed when straightly considering the methodology proposed in [14]: no single species abundantly occurs in pre- and post-flame regions as the $\mathrm{N}_{2}$ in air-blown combustion to be used as a "filling gas". Therefore, in the present investigations the surrounding gas mixture is simplified by a couple of major species, in which their molar fractions are re-scaled according to

$$
X_{i}^{*}=X_{i}\left(\frac{X_{\text {major }}}{\sum_{j}^{M} X_{j}}\right), \quad \text { with } \quad X_{\text {major }}=1-X_{\text {vapor }} \quad \text { and } \quad j=1,2, \ldots, M,
$$

where $M$ is the total number and $j$ is the list index of the selected major species.

\section{Results and discussion}

The evaluation of the carrier gas modeling is structured in three approaches: (A1) detailed description (reference), (A2) combination of selected major species, and (A3) pure air. This evaluation is conducted in three different scenarios, which are defined by the oxidizer composition: (S1) pure air, (S2) pure $\mathrm{O}_{2}$, and (S3) a mixture of $50 \% \mathrm{O}_{2}$ and $50 \% \mathrm{~N}_{2}$ in volume. In oxy-fuel scenarios (i.e. S2 and S3), flames propagating in droplet mists are considered within an equivalence ratio $(\phi)$ range of $[0.6,1.4]$, while in air-blown this range is reduced to $[0.9,1.4]$. For all scenarios, droplets are injected with an initial diameter of $40 \mu \mathrm{m}$ at $3.0 \mathrm{~cm}$ upstream of the reaction zone, which is in agreement with [10] as well as the definition of the scenario S3. In order to select the major species used to calculate the carrier gas properties $\mu, \lambda, c_{p}$, and $D_{i j}$, the procedure presented in [14] is applied on the reference results associated with each scenario. Namely, simulations conducted with a detailed description of carrier gas composition, for a mixture composition within the corresponding $\phi$ range, and droplet initial diameter of 75 $\mu \mathrm{m}$ (in agreement with [14]). Table 1 summarizes the ten species of highest mass fractions along the corresponding droplets lifetimes for each scenario. It is important to mention that the evaluation of the impact of species combination on specific properties (i.e. $\mu, \lambda, c_{p}$, and $D_{i j}$ ) as done in [14] is subject of future works. In the present study, the impact of different modeling strategies for the carrier gas are only evaluated in terms of the flame propagation speed. However, the know-how developed in [14] is embedded in many choices and decisions taken throughout this study, for instance in the choice of common major species to all scenarios.

Table 1. Major species ranking list by oxidizer specification (Ox.).

\begin{tabular}{l|llllllllll}
\hline Ox. $\backslash$ Rank. & 1 & 2 & 3 & 4 & 5 & 6 & 7 & 8 & 9 & 10 \\
\hline S1 & $\mathrm{N}_{2}$ & $\mathrm{CO}_{2}$ & $\mathrm{O}_{2}$ & $\mathrm{CO}$ & $\mathrm{CH}_{4}$ & $\mathrm{H}_{2}$ & $\mathrm{OH}$ & $\mathrm{O}$ & $\mathrm{NO}$ & $\mathrm{H}$ \\
S2 & $\mathrm{CO}_{2}$ & $\mathrm{O}_{2}$ & $\mathrm{CO}$ & $\mathrm{OH}$ & $\mathrm{O}$ & $\mathrm{H}_{2}$ & $\mathrm{CH}_{4}$ & $\mathrm{H}$ & $\mathrm{HO}_{2}$ & $\mathrm{H}_{2} \mathrm{O}_{2}$ \\
S3 & $\mathrm{N}_{2}$ & $\mathrm{CO}_{2}$ & $\mathrm{O}_{2}$ & $\mathrm{CO}$ & $\mathrm{OH}$ & $\mathrm{NO}$ & $\mathrm{O}$ & $\mathrm{H}_{2}$ & $\mathrm{CH}_{4}$ & $\mathrm{H}$ \\
\hline
\end{tabular}

From the results presented in Table 1, a common major species combination is proposed to represent the different investigated scenarios. Accordingly, the combination $\mathrm{CO}_{2}+\mathrm{O}_{2}+\mathrm{CO}+$ $\mathrm{OH}+\mathrm{H}_{2}+\mathrm{CH}_{4}$ has been chosen, hereafter denoted by MC. This choice was based on the occurrence of such species in all investigated scenarios, while preserving a minimum number of two species to describe both pre- and post-flame regions. The inclusion of $\mathrm{H}_{2}$ in the $\mathrm{MC}$ combination follows the great contribution of such species in properties prediction perceived in [14]. Particularly, this low molecular weight interferes in the adopted selection of major species, 

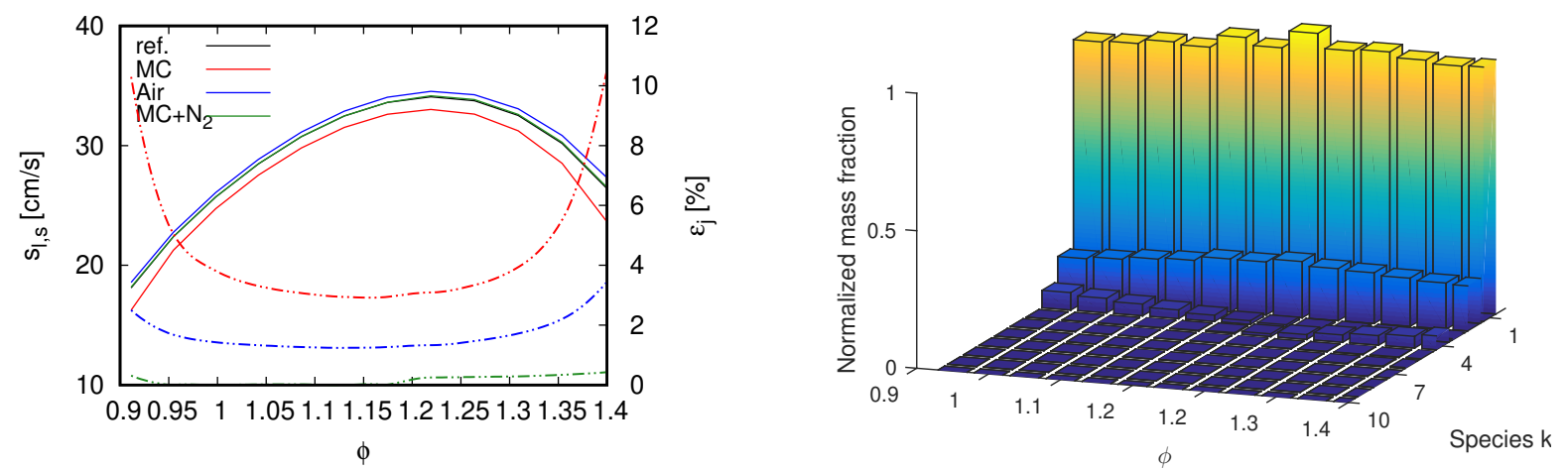

Figure 1. Air-blown scenario (S1). Left: $s_{l, s}$ (continuous line) and $\varepsilon_{i}$ (dash-dot line) values for different mixture compositions. Right: normalized mass fractions of major species following the ranking presented in Table 1.
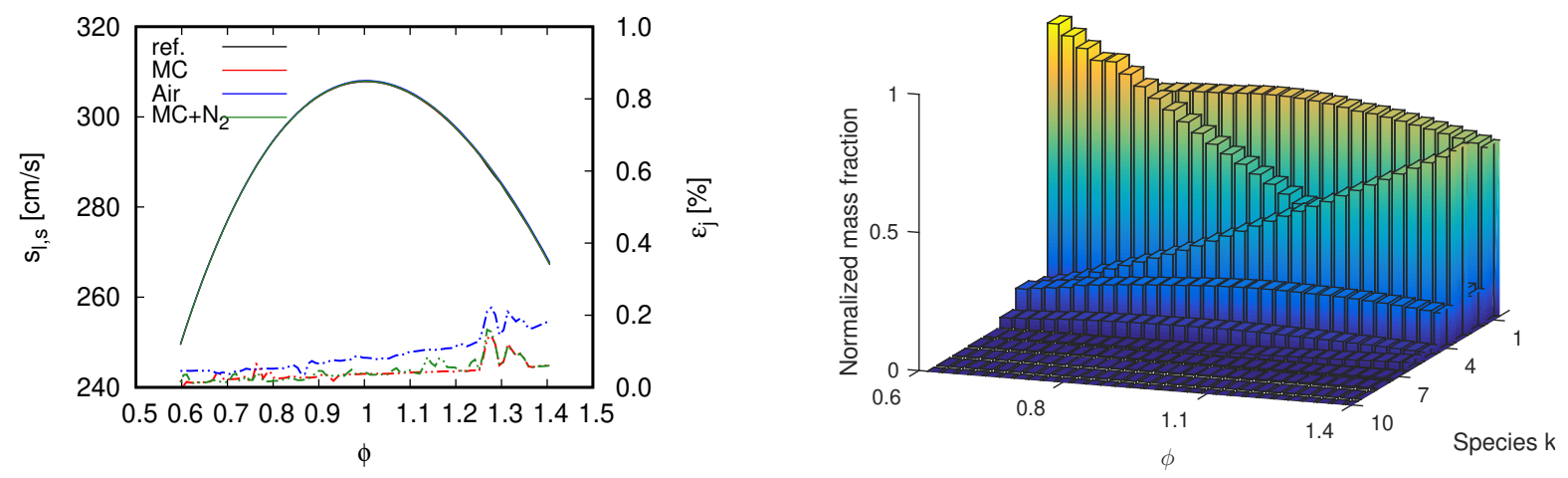

Figure 2. Pure oxy-fuel scenario (S2). Left: $s_{l, s}$ (continuous line) and $\varepsilon_{i}$ (dash-dot line) values for different mixture compositions. Right: normalized mass fractions of major species following the ranking presented in Table 1.
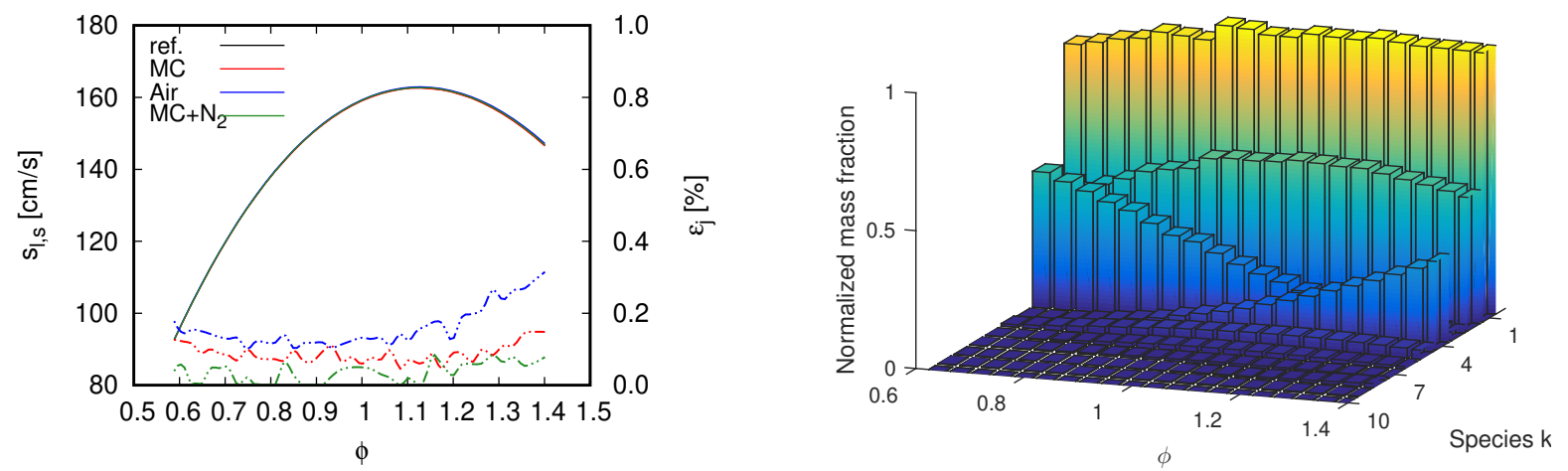

Figure 3. Scenario with an oxidizer composition of $50 \% \mathrm{O}_{2}$ and $50 \% \mathrm{~N}_{2}$ (S3). Left: $s_{l, s}$ (continuous line) and $\varepsilon_{i}$ (dash-dot line) values for different mixture compositions. Right: normalized mass fractions of major species following the ranking presented in Table 1.

which is based on mass fractions.

Figures 1-3 presents values of the laminar flame speed $\left(s_{l, s}\right)$ for different mixture compositions and the normalized mass fraction $(\bar{Y})$ of major species $k$ respectively for the scenarios with oxidizer composition of pure air (S1), pure $\mathrm{O}_{2}$ (S2), and $50 \% \mathrm{O}_{2}$ and $50 \% \mathrm{~N}_{2}$ (S3). Additionally, in flame speed plots, deviations for each tested simplification case $\left(\varepsilon_{j}-j\right.$ is the index of a specific simplification case) to the corresponding reference are included. On the right side of Figs. 1-3, the normalized mass fractions of major species following the ranking presented in Table 1 are exhibited. These bar plots allow to identify the relative importance, in terms of mass fractions, of each selected major species for the computation of the carrier gas properties. 
By comparing the different carrier gas simplifications in each scenario, it turns out that the simplification of the carrier gas as pure air delivers a good agreement with the reference case in all scenarios. This outcome was not expected in the oxy-fuel combustion scenario where the oxidizer is composed of pure $\mathrm{O}_{2}$. However, some care must be taken on the interpretation of such results. To obtain a general performance of such a simplification to oxy-fuel combustion diluted with liquid water, variations in mists characteristics must be accounted for.

The simplification of the carrier gas mixture by a combination of common species to all the different scenarios demonstrated to be insufficient as a universal combination to such scenarios. Within the so-called MC combination, $\mathrm{N}_{2}$ is not present, since for pure oxy-fuel combustion this species is absent. Accordingly, the largest deviations found when applying the MC combination occurs for the air-blown combustion scenario. The high amount of $\mathrm{N}_{2}$ in S3 leads to the interpretation that the absence of this species would also significantly interfere with the performance of the MC combination. However, this does not occur. As seen in Fig. 3 deviations lower than $0.4 \%$ are noticed.

The good agreement of the MC combination to the reference cases for both oxy-fuel combustion scenarios (S2 and S3), or the deviations found between MC combination and reference cases for air-blown combustion, can be explained by the relative importance of $\mathrm{N}_{2}$ in such scenarios. This information can be appraised by means of the normalized species mass fraction plots on the right side of Figs. 1-3. By comparing the $\bar{Y}$ values of the first two most predominant major species in both cases where $\mathrm{N}_{2}$ is present, i.e. $\mathrm{N}_{2}$ and $\mathrm{CO}_{2}$, the relative importance of $\mathrm{N}_{2}$ is much higher for air-blown than for the $\mathrm{S} 3$ in the entire range of $\phi$. This influences the computation of properties necessary to the modeling of the evaporation process, which is consequently reflected in the values of $s_{l, s}$ presented in Figs. 1 and 3 . As follows, the inclusion of $\mathrm{N}_{2}$ in $\mathrm{MC}$ combination (see $\mathrm{MC}+\mathrm{N}_{2}$ results in Figs. 1-3) demonstrated to be unnecessary in oxy-fuel combustion scenarios, while the opposite occurs for air-blown cases.

A specific aspect related to the normalized mass fractions plot refers to the correspondence of major species rank for oxy-fuel combustion scenarios (S2 and S3) and the evolution of such mass fractions throughout the equivalence ratio range. Except by $\mathrm{N}_{2}$ the four most predominant major species in both scenarios are the same. But not only this, the trends of $\bar{Y}$ of such species are quite similar in Figs. 2 and 3 . Regarding the air-blown combustion scenario, a deviation occurs comparing it with both oxy-fuel combustion ones. Herein, the ranking position of $\mathrm{O}_{2}$ and $\mathrm{CO}$ are inverted. Although such an inversion is reflected in $\bar{Y}$ profiles, their trends throughout the equivalence ratio range are preserved.

As a summary for the evaluation of carrier gas simplifications, the results presented in Figs. 1-3 indicate that the MC combination is sufficient for oxy-fuel combustion scenarios. Such an outcome is reinforced considering the fact that despite being utilized (e.g. [3, 7, 21])), high amounts of $\mathrm{N}_{2}$ in the oxidizer composition are not desired in oxy-fuel combustion dedicated to CCS technologies. However, for air-blown combustion, $\mathrm{N}_{2}$ should be accounted for in species combinations dedicated to the carrier gas modeling.

With respect to the overall behavior of results presented in Figs. 1-3, it is noticed that flames propagating in water droplet mists depict the bell shape curve typically observed in freely propagating gas flames. Such a behavior contrasts with the results achieved in [15] for flames propagating in fuel droplet mists and occurs here in all tested scenarios. Regarding the air blown combustion, the maximum value of the flame speed is clearly found within the rich part of the mixture composition range when water dilution occurs. As previously seen in [10], this behavior is intensified when water dilution increases. Such a behavior can be explained by the fact that the diluent agent is found in the oxidizer stream and lean mixtures have therefore higher values of liquid water. As a result, the latent heat consumed to promote the evaporation process is higher in lean than in rich flames, which consequently lowers the flame speed values. In this sense, the depletion of $\mathrm{N}_{2}$ in the oxidizer stream attenuates this shifting, which is observed by comparing Figs. 1-3. 


\section{Conclusions}

A novel modeling simplification approach applied to represent the surrounding gas composition of water droplets in air-blown and oxy-fuel combustion atmospheres is proposed and validated. Along with this study, an analysis of other simplifications approaches is also presented. The entire study is conducted within the context of one-dimensional numerical simulations of flames propagating in droplet mists considering a detailed description of the chemistry.

Simulations results clearly indicate that the simplification of the carrier gas composition by a combination of common major species to air-blown and oxy-fuel combustion is not the best option for all scenarios. As nitrogen is absent in such a combination, flame speed values in airblown scenario are underestimated. In this sense, the proposed MC combination is an accurate choice for general oxy-fuel combustion applications while for air-blown combustion $\mathrm{N}_{2}$ must be added to this species combination. For the investigated scenarios and mists characteristics, the simplification of the carrier gas composition by air delivered flame speed values with a reasonable agreement to the reference cases in all scenarios. However, to obtain a general performance of such a simplification to oxy-fuel combustion further analysis is needed.

The performance of the simplifications presented in this manuscript were evaluated in terms of flame speed values. Detailed analysis and assessment of minimal species combinations as done for flames propagating in fuel droplets in [14] are subject of future work. Additionally, this study can be extended including variations in droplet mist characteristics and in oxidizer composition of flames propagating in fuel droplet mists to allow a general assessment of simplifications in air-blown and oxy-fuel combustion.

\section{Acknowledgements}

We gratefully acknowledge support of the RCGI - Research Centre for Gas Innovation, hosted by the University of São Paulo (USP) and sponsored by FAPESP - São Paulo Research Foundation (2014/50279-4) and Shell Brasil, and the strategic importance of the support given by ANP (Brazil's National Oil, Natural Gas and Biofuels Agency) through the R\&D levy regulation.

\section{References}

[1] B. Abramzon and W. A. Sirignano. Droplet vaporization model for spray combustion calculations. Int. J. Heat Mass Transf., 32(9):1605-1618, 1989.

[2] A. Both. RANS-FGM simulation of $n$-heptane spray flame in OpenFOAM: A new implementation of flamelet generated manifold to account for enthalpy loss with detailed reaction mechanisms. PhD thesis, Delft University of Technology, Delft, The Netherlands, 2017.

[3] M. Ditaranto and J. Bakken. Study of a full scale oxy-fuel cement rotary kiln. Int. J. Greenh. Gas Control, 83:166-175, apr 2019.

[4] A. Ern and V. Giovangigli. Fast and Accurate Multicomponent Transport Property Evaluation. J. Comput. Phys., 120(1):105-116, aug 1995.

[5] B. Franzelli, A. Vié, M. Boileau, B. Fiorina, and N. Darabiha. Large Eddy Simulation of Swirled Spray Flame Using Detailed and Tabulated Chemical Descriptions. Flow, Turbul. Combust., 98(2):633-661, sep 2017.

[6] L. Ma, B. Naud, and D. Roekaerts. Transported PDF Modeling of Ethanol Spray in HotDiluted Coflow Flame. Flow, Turbul. Combust., 96(2):469-502, mar 2016.

[7] A. N. Mazas, D. A. Lacoste, and T. Schuller. Experimental and numerical investigation on the laminar flame speed of $\mathrm{CH} 4 / \mathrm{O} 2$ mixtures diluted with $\mathrm{CO} 2$ and $\mathrm{H} 2 \mathrm{O}$. In Proc. ASME 
Turbo Expo, volume 2, pages 411-421. American Society of Mechanical Engineers Digital Collection, dec 2010.

[8] R. S. Miller, K. Harstad, and J. Bellan. Evaluation of equilibrium and non-equilibrium evaporation models for many-droplet gas-liquid flow simulations. Int. J. Multiph. Flow, 24(6):1025-1055, sep 1998.

[9] A. Neophytou and E. Mastorakos. Simulations of laminar flame propagation in droplet mists. Combust. Flame, 156(8):1627-1640, aug 2009.

[10] F. L. Sacomano Filho, L. E. de Albuquerque Paixão e Freire de Carvalho, J. A. van Oijen, and G. C. Krieger Filho. Effects of Reaction Mechanisms and Differential Diffusion in Oxy-Fuel Combustion Including Liquid Water Dilution. Fluids, 6(2):47, jan 2021.

[11] F. L. Sacomano Filho, L. Dressler, A. Hosseinzadeh, A. Sadiki, and G. C. Krieger Filho. Investigations of evaporative cooling and turbulence flame interaction modeling in ethanol turbulent spray combustion using tabulated chemistry. Fluids, 4(4):187, oct 2019.

[12] F. L. Sacomano Filho, A. Hosseinzadeh, A. Sadiki, and J. Janicka. On the interaction between turbulence and ethanol spray combustion using a dynamic wrinkling model coupled with tabulated chemistry. Combust. Flame, 215:203-220, may 2020.

[13] F. L. Sacomano Filho, G. C. Krieger Filho, J. A. van Oijen, and A. Sadiki. Numerical investigation of droplet evaporation modeling in combustion environment. In ICLASS 2018 - 14th Int. Conf. Liq. At. Spray Syst., page 8, Chicago, 2020.

[14] F. L. Sacomano Filho, G. C. Krieger Filho, J. A. van Oijen, A. Sadiki, and J. Janicka. A novel strategy to accurately represent the carrier gas properties of droplets evaporating in a combustion environment. Int. J. Heat Mass Transf., 137:1141-1153, jul 2019.

[15] F. L. Sacomano Filho, N. Speelman, J. A. van Oijen, L. P. H. de Goey, A. Sadiki, and J. Janicka. Numerical analyses of laminar flames propagating in droplet mists using detailed and tabulated chemistry. Combust. Theory Model., 22(5):998-1032, 2018.

[16] P. S. Sánchez. Modeling the dispersion and evaporation of sprays in aeronautical combustion chambers 3. Phd thesis, Institut National Polytechnique de Toulouse - INPT, Toulouse, France, 2012.

[17] S. S. Sazhin. Advanced models of fuel droplet heating and evaporation. Prog. Energy Combust. Sci., 32(2):162-214, 2006.

[18] W. A. Sirignano. Fluid Dynamics and transport of droplets and sprays. Cambridge University Press, New York, NY, 2nd edition, 2010.

[19] B. Somers. The simulation of flat flames with detailed and reduced chemical models. PhD thesis, Technische Universiteit Eindhoven, 1994.

[20] M. C. Yuen and L. W. Chen. On Drag of Evaporating Liquid Droplets. Combust. Sci. Technol., 14(4-6):147-154, oct 1976.

[21] C. Zheng and Z. Liu. Oxy-fuel combustion: Fundamentals, theory and practice. Elsevier, jan 2017. 